\title{
A comparative study of single Theileria lestoquardi and mixed infections with Theileria ovis
}

\author{
Salama Al-Hamidhi', Elshafie I. Elshafie ${ }^{2,3}$, Saeed Yaghfoori ${ }^{4}$, W. Ivan Morrison ${ }^{5}$, Eugene H. Johnson ${ }^{2}$ and \\ Hamza A. Babiker ${ }^{1,6^{*}}$
}

\begin{abstract}
Background: Epidemiological surveys in Oman have revealed a high prevalence of the co-occurrence of the pathogenic Theileria lestoquardi and the non-pathogenic Theileria ovis among sheep in the Barka region, Oman. Our most recent data illustrated an interaction and reduced mortality risk in animals co-infected with T. lestoquardi and T. ovis, suggesting that the latter confers protection against pathogenicity of T. lestoquardi. The present study extends the above findings and examines disease outcomes; clinical markers, hematological parameters, and parasite density in mixed and single T. lestoquardi infections.
\end{abstract}

Methods: A total of 390 blood samples were collected from 16 sheep pens located in Barka, Oman between July and November 2019. Theileria spp. were detected and quantified using qPCR assay targeting 185 rRNA, and the extent of genetic diversity was estimated by a panel of T. lestoquardi specific micro- and mini-satellites. The association of some disease markers with the presence of Theileria spp. and genetic diversity was tested.

Results: Theileria spp. were detected in 75 (19.2\%) sheep; of these 65 (86.7\%) had mixed infections (T. lestoquardi plus T. ovis), 8 (10.6\%) were infected with T. lestoquardi alone, and 2 (2.7\%) with only T. ovis. Exotic breeds had a higher risk for Theileria spp. infection. The density (18S rRNA gene copies) of both parasites was higher in single infection against mixed infection, and there was a relatively lower density of T. lestoquardi in mixed infections. However, there was no difference in hematological indices between single T. lestoquardi and mixed infections. High genetic diversity was observed among T. lestoquardi in Barka, with no differences of T. lestoquardi in single and mixed infections. The extent of diversity seen in Barka was higher $(\mathrm{He}=0.772)$ than that reported in Oman in 2019 ( $\mathrm{He}=0.582)$, with distinct T. lestoquardi genotypes.

Conclusion: The lower density of T. lestoquardi as mixed infection with T. ovis compared to single infection supports the hypothesis that T. ovis confers protection against lethal T. lestoquardi infection. However, there were no differences in disease correlations (clinical markers, hematological parameters, and density of parasites) or the extent of diversity of T. lestoquardi between the two types of infection. The presence of distinct T. lestoquardi genotypes in Barka, compared to that reported earlier in Oman, likely reflects movement of carrier animals and highlights the need for further analysis of the parasite populations to inform novel approaches for controlling malignant ovine theileriosis.

Keywords: Theileriosis, Theileria lestoquardi, T. ovis, Genetic diversity

\footnotetext{
*Correspondence: h.babiker@ed.ac.uk

†Salam Al-Hamidhi and Elshafie I Elshafie are equal contribution to the study.

${ }^{1}$ Department of Biochemistry, College of Medicine and Health Sciences,

Sultan Qaboos University, AlKhoud 123, PO Box 35, Muscat, Oman

Full list of author information is available at the end of the article
}

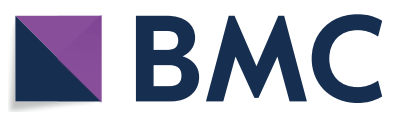

(c) The Author(s) 2021. Open Access This article is licensed under a Creative Commons Attribution 4.0 International License, which permits use, sharing, adaptation, distribution and reproduction in any medium or format, as long as you give appropriate credit to the original author(s) and the source, provide a link to the Creative Commons licence, and indicate if changes were made. The images or other third party material in this article are included in the article's Creative Commons licence, unless indicated otherwise in a credit line to the material. If material is not included in the article's Creative Commons licence and your intended use is not permitted by statutory regulation or exceeds the permitted use, you will need to obtain permission directly from the copyright holder. To view a copy of this licence, visit http://creativecommons.org/licenses/by/4.0/. The Creative Commons Public Domain Dedication waiver (http://creativeco mmons.org/publicdomain/zero/1.0/) applies to the data made available in this article, unless otherwise stated in a credit line to the data. 


\section{Background}

Malignant ovine theileriosis is an important hemoprotozoal disease of sheep and goats in tropical and subtropical regions caused by pathogenic Theileria spp. including T. lestoquardi, T. luwenshuni and T. uilenbergi $[1,2]$. In addition, there is frequent infection of small ruminants with non-pathogenic Theileria spp., including T. ovis, T. separata, and $T$. recondite $[1,2]$. In China, T. uilenbergi and T. luwenshuni are highly pathogenic, especially in sheep, and cause severe clinical signs [3]. Theileria lestoquardi is among the most common and pathogenic Theileria spp. of small ruminants in the Arabian Peninsula and is highly prevalent in the Gulf Cooperation Council (GCC) countries, causing high morbidity and mortality among indigenous sheep [4]. Recovery from acute infection following chemotherapy is often followed by persistence of some parasites-referred to as the carrier state-which is often undetectable microscopically but enables transmission by the tick vector $[5,6]$. The carrier state creates a significant risk for the spread of disease into other theileriosis-free areas where the vector is present. Chronic asymptomatic carriage of Theileria spp. has been linked to significant losses in livestock productivity [7].

Previous surveys in Oman have demonstrated high rates of asymptomatic infection with Theileria spp. among cattle (72.3\%) and sheep (37.5\%) [8]. Interestingly, sheep frequently had mixed infections with T. lestoquardi (pathogenic) and T. ovis (non-pathogenic) [5, 9], which are both transmitted by Hyalomma anatolicum, the predominant tick species identified on animals examined in Oman [5]. Our most recent data illustrated a potential competitive interaction between these two Theileria spp.; a substantial reduction in the risk of mortality due to $T$. lestoquardi was observed in mixed-parasite infections, suggesting that $T$. ovis confers protection against the pathogenic effect of $T$. lestoquardi infection [10]. A protective effect of mixed-species infection (T. lestoquardi $+T$. ovis) against severe malignant ovine theileriosis (MOT), is in line with a large body of data on apicomplexan parasites indicating that mixed infections with different species may modulate the virulence and pathogenicity of each other [11]. Evidence of a crossspecies protective effect has been shown in a number of apicomplexan parasites, such as the reciprocal effect between the bacterium Anaplasma phagocytophilum and Babesia microti parasite in field voles [12], and the suppression of $B$. divergens by $A$. phagocytophilum in cattle [13]. More recently, a field epidemiological study in indigenous zebu calves in East Africa demonstrated an $89 \%$ reduction in mortality due to $T$. parva infection (East Coast fever) in the presence of less pathogenic Theileria spp. (T. mutans and T. velifera) [14]. Understanding the consequences of mixed infections to variations in Theileria spp. pathogenicity is critical for designing control measures aimed at reducing morbidity and mortality.

Infections with Theileria parasites induce a range of hematologic and biochemical changes associated with the intra-leukocyte schizont and intra-erythrocyte piroplasm stages. The severity of these changes is related to the virulence of the strain, the infectious dose, the animal breed, and immune status [15-17]. Experimental infections of cattle and sheep, respectively, with $T$. annulata and T. lestoquardi, have demonstrated a significant progressive decrease in the concentration of hemoglobin, hematocrit, and red blood cells, as well as leukopenia and leukocytolysis [18-20]. The absence of hemoglobinemia indicates that the decrease in hematocrit and red blood cells may be due to erythrocyte destruction by mononuclear phagocyte system rather than intravascular lysis $[17,20]$.

The present study extends our previous findings in Oman by examining clinical and hematological indices in sheep naturally co-infected with $T$. lestoquardi and $T$. ovis to determine whether interactions between the pathogenic and less pathogenic species of Theileria significantly reduces the impact of theileriosis. Such information can indicate whether infection of the nonpathogenic T. ovis can be utilized as a predictor of clinical outcome of MOT. In addition, we examined the influence of genetic complexity on disease indices and compared the extent of diversity of $T$. lestoquardi in the current study to that collected in other regions in 2014, to investigate temporal changes in parasite structure in Oman.

\section{Methods}

\section{Study area and sampling}

A cross-sectional study was conducted in Barka (Al-Bāținah South Governorate, Fig. 1) between July and November 2019. A total of 390 blood samples were collected from 16 sheep farms, number of examined animals per farm ranged between 10 to 56 . The examined animals include indigenous breed, North of Oman breed, and exotic, imported from Saudi Arabia, Somalia, India, and Iran. Some of the screened farms had a history of theileriosis. Lymph node palpation, rectal temperature, and the color of the mucus membranes of the eyes were assessed during sample collection. In addition, the age, sex, and breed of the animals were recorded. The study area is characterized by a hot climate with a long summer season from mid of April to October. The temperature can reach up to $50.8^{\circ} \mathrm{C}$. Characteristically, there is only a small amount of rainfall throughout the year with $100 \mathrm{~mm}$ average annual rainfall, mostly in January. Animals are kept outdoors, in secured enclosures, due to a lack of open natural 


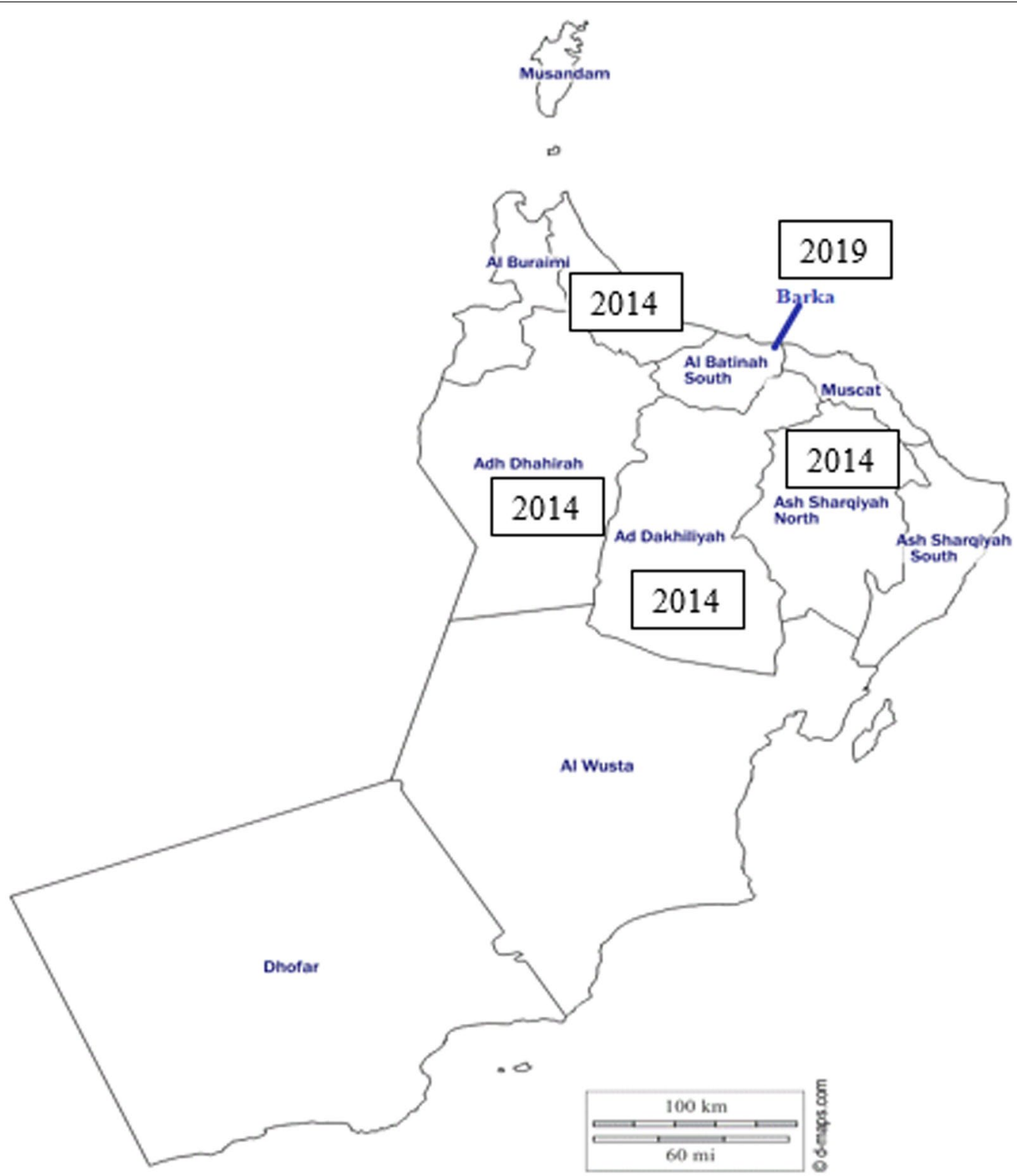

Fig. 1 Locations of collection sites from Barka, Al-Bāținah South Governorate (2019) and other regions in Oman (2014), Al-Bāținah, Adh Dhahirah, Ad Dakhiliya, As Sharqoyah, Dhofar. 2014 and 2019 refer to study years when samples were collected

pastures, and fed on Rhodes grass hay. They are herded into shelters; however, movement from farm to farm is not an uncommon practice.

The research was performed in accordance with the relevant guidelines and regulations of the ethical and animal welfare code of Sultan Qaboos University, Oman and the Ministry of Agriculture and Fisheries, Oman. Oral consent was obtained from the farm owner before drawing blood from animals.

\section{Hematological assessment}

Five milliliters of blood was collected from individual animals via venipuncture into Vacutainer tubes containing EDTA and immediately transported to the laboratory in iceboxes for determination of hematological parameters and DNA extraction.

Hematological parameters including total erythrocyte count (RBC), hematocrit (HCT), hemoglobin concentration $(\mathrm{Hb})$, mean corpuscular volume $(\mathrm{MCV})$, mean 
corpuscular hemoglobin concentration (MCHC), and total white blood cell count (WBC), as well as differentials, were determined using an Exigo H400 veterinary hematology analyzer (Spånga, Sweden).

\section{Detection and quantification of $T$. lestoquardi and $T$. ovis} DNA was extracted from $200 \mu \mathrm{l}$ of blood, using the QIAamp DNA mini kit (Qiagen, Germany) according to the manufacture's instruction, and stored at $-20{ }^{\circ} \mathrm{C}$. Theileria spp. were identified by sequencing polymerase chain reaction (PCR) products obtained using pan-Theileria primers, targeting the $18 S$ rRNA as described elsewhere [21].

The density of T. lestoquardi and T. ovis parasites was quantified using real-time polymerase chain reaction (qPCR) to determine the gene copy number. Speciesspecific primers and probes targeting the 18S rRNA gene of T. lestoquardi and T. ovis were used; details of assay optimization are described elsewhere [10]. Standard curves were generated using quantified DNA of T. lestoquardi $\left(9.26 \times 10^{10} 18 S r R N A\right.$ gene copies/ $\left.\mu \mathrm{L}\right)$ and $T$. ovis $\left(5.3 \times 10^{10} 18 S r R N A\right.$ gene copies $\left./ \mu \mathrm{L}\right)$.

\section{Typing of $T$. lestoquardi microsatellites}

T. lestoquardi was genotyped using a panel of ten unlinked polymorphic microsatellite DNA markers as described elsewhere [9]. Labeled PCR products were mixed with Gene-Scan ${ }^{\mathrm{TM}} 500$ ROX internal size standard (Applied Biosystems, UK) for capillary electrophoresis on an ABI 3130XL Genetic Analyzer (Applied Biosystems, UK). GeneMapper software version 4 (Applied Biosystems, UK) was used for scoring allele sizes and quantifying electropherogram peak heights for samples containing multiple alleles per locus. Multiple alleles per locus were scored if electrophoretic peaks corresponding to minor alleles were $>32 \%$ of the height of the predominant allele [22].

\section{Data analysis}

Data as mean and standard deviation for hematological parameters were compared among the fixed factors (sheep infected with T. lestoquardi alone, mixed infection "T. lestoquardi and T. ovis", and uninfected group). For this purpose, multivariate analysis of covariance by controlling for potential confounding factors such as age, sex, breed, and farm was performed. Hematological parameters, including WBCs, differential WBCs, and platelets, were log-transformed to meet the assumption of normality. Categorical responses were expressed as a percentage, and the association of potential risk factors such as sex, age, breed, presence of ticks, enlargement of lymph nodes, and pallor of ocular mucus membrane were tested individually using Pearson's chi-squared or Fisher's exact test using univariate logistic regression (Table 1). The model of binary logistic regression was performed using the backward stepwise method to test the significance of the variables tested by the univariate analysis (Table 2). Odds ratio and respective $95 \%$ confidence interval (CI) were also calculated for the significant variables. Odds ratio with significant result and value less than one were inversed to facilitate interpretation. The effect of parasite density on hematology indices was assessed using regression method. All analyses were done with SPSS Statistical Software for Windows, version 24.0 (IBM Corp., Armonk, NY, USA). All tests were twotailed, and $P$-values of less than 0.05 were considered statistically significant.

Microsatellite allele data were filtered to retain only minor alleles having a peak height of $>32 \%$ of the corresponding predominant alleles if more than one allele was present at any given locus. Genetic diversity metrics were calculated for the entire dataset using GenAlEx 6.5 [23]. Expected heterozygosity was calculated using the formula for "unbiased heterozygosity," also termed haploid genetic diversity, $\mathrm{He}=[n /(n-1)]\left[1-\sum p 2\right]$, where $n$ is the number of isolates and $p$ the frequency of each different allele at a given locus [24]. Population differentiation was assessed by estimating Wright's FST index using the FSTAT computer program version 2.9.3.2. Two estimators of FST (G'ST and $\theta$ ) [25] were used to estimate genetic differentiation between imported parasites from the Indian subcontinent and Africa.

Multiplicity of infection (MOI), defined as the presence of multiple genotypes per infection, was determined by the detection of more than one allele at a given locus. To avoid over-estimation of low-abundance alleles, only minor alleles having a peak height of $>33 \%$ (one third) of the corresponding predominant alleles were accepted. The proportion of samples with more than one allele across ten loci was used to represent MOI.

Variance of mismatch values (VD) were compared to values of $\mathrm{L}$ (the upper confidence limits of Monte Carlo simulation and parametric tests), and where $\mathrm{VD}>\mathrm{L}$ linkage disequilibrium is assumed [26].

\section{Results \\ Demographic data}

A total of 390 sheep, comprising three breeds, indigenous $(n=291)$, exotic $(n=75)$, and crossbreeds $(n=24)$, were screened for the presence of $T$. lestoquardi by PCR assay of $18 S$ rRNA (Table 1). The majority of sheep 212 (54.4\%) were infested with adult ticks, and 57 (14.6\%) had elevated rectal temperatures $>40{ }^{\circ} \mathrm{C}$. Enlargement of lymph nodes was observed in 255 sheep (65.4\%), either unilaterally 
Table 1 Univariate analysis for the association of potential risk factors with Theileria sp. infection among sheep in Barka, Oman

\begin{tabular}{|c|c|c|c|c|c|}
\hline \multirow[t]{2}{*}{ Variables } & \multirow[t]{2}{*}{ Number (\%) } & \multicolumn{2}{|l|}{$P C R$} & \multirow[t]{2}{*}{ Chi-square test } & \multirow[t]{2}{*}{$P$-value } \\
\hline & & $\begin{array}{l}\text { Positive } \\
n=75 \text { (19.2\%) }\end{array}$ & $\begin{array}{l}\text { Negative } \\
n=315 \text { (80.8\%) }\end{array}$ & & \\
\hline \multicolumn{6}{|l|}{ Sex } \\
\hline Male & $64(16.4)$ & $18(28.1)$ & $46(71.9)$ & 3.90 & 0.05 \\
\hline Female & $326(83.6)$ & $57(17.5)$ & $269(82.5)$ & & \\
\hline \multicolumn{6}{|l|}{ Age } \\
\hline Less than 1 year & $122(31.3)$ & 19 (15.6) & $103(84.4)$ & 3.02 & 0.22 \\
\hline $1-2$ years & $106(27.2)$ & $26(24.5)$ & $80(75.5)$ & & \\
\hline More than 2 years & $162(41.5)$ & $30(18.5)$ & $132(81.5)$ & & \\
\hline \multicolumn{6}{|l|}{ Breed $^{\mathrm{a}}$} \\
\hline Exotic & $75(19.2)$ & $27(36)$ & $48(64)$ & 18.86 & 0.001 \\
\hline Cross-bred & $24(6.2)$ & $1(4.2)$ & $23(95.8)$ & & \\
\hline Indigenous & 291(64.6) & $47(16.2)$ & $244(83.8)$ & & \\
\hline \multicolumn{6}{|l|}{ Presence of ticks } \\
\hline Yes & $178(45.6)$ & $37(20.8)$ & $141(79.2)$ & 0.51 & 0.48 \\
\hline No & $212(54.4)$ & $38(17.9)$ & $174(82.1)$ & & \\
\hline \multicolumn{6}{|l|}{ Lymph node } \\
\hline Normal & $135(34.6)$ & $30(22.2)$ & $105(77.8)$ & 1.19 & 0.28 \\
\hline Enlarged & $255(65.4)$ & $45(17.6)$ & $210(82.4)$ & & \\
\hline \multicolumn{6}{|c|}{ Mucous membrane color } \\
\hline Normal & $334(85.6)$ & $63(18.9)$ & $271(81.1)$ & 0.20 & 0.65 \\
\hline Pale & $56(14.4)$ & $12(21.4)$ & 44 (78.6) & & \\
\hline
\end{tabular}

${ }^{a}$ Significant difference

Table 2 Model of binary logistic regression using backward stepwise method of the hypothesized risk factors with the frequency of Theileria sp. infection

\begin{tabular}{llllll}
\hline Variables & $\beta$ & SE $\beta$ & P-value & Adjusted odds ratio & $\frac{95 \% \mathrm{Cl}}{\text { Lower }}$ \\
\hline Breed & & & & - \\
Exotic & 0.00 & - & - & 1 & 0.01 \\
Cross-bred & -2.52 & 1.05 & 0.016 & 0.08 & 0.63 \\
Indigenous & -1.05 & 0.30 & 0.001 & 0.35 & 0.19 \\
\hline
\end{tabular}

$\beta$ : logistic coefficients. SE: standard error, $\mathrm{Cl}$ : confidence interval. Odds ratio of sheep breed: exotic sheep were 2.9 (CI 1.66-5.14) and 12.9 (Cl 1.65-101.19) times as likely to be Theileria-positive as indigenous and cross-bred sheep, respectively

(32.6\%, $n=127)$ or bilaterally $(32.8 \%, n=128)$. In addition, pale mucus membrane was recorded in 56 animals (14.4\%).

Theileria spp. were detected in 75 (19.2\%) of the examined animals; 8 (10.6\%) carried single T. lestoquardi, 2 (2.7\%) had single T. ovis, and 65 (86.7\%) harbored mixed-species infections with T. lestoquardi and T. ovis. There was a great variation in the prevalence of
Theileria spp., in the examined farms, ranging between $5.9 \%$ and $53.5 \%$.

\section{Clinical and hematological indices among sheep with different Theileria species}

Theileria spp. prevalence was not associated with age $(p=0.22)$ or sex $(p=0.05)$ of the animals across the region. Similarly, no differences were observed between 
Table 3 Multivariate analysis of covariance for hematological indices among sheep infected with T. lestoquardi alone, mixed infection (T. lestoquardi and T. ovis), and uninfected groups

\begin{tabular}{|c|c|c|c|c|c|c|}
\hline \multirow[t]{2}{*}{ Blood indices } & \multirow[t]{2}{*}{ Reference range } & \multicolumn{3}{|l|}{$\begin{array}{l}\text { Groups } \\
\text { Mean } \pm S D\end{array}$} & \multirow[t]{2}{*}{$F$ test } & \multirow[t]{2}{*}{$P$-value } \\
\hline & & $\begin{array}{l}\text { Tlestoquardi } \\
\mathrm{n}=8(2.1 \%)\end{array}$ & $\begin{array}{l}\text { Mixed infection } \\
n=65(16.8 \%)\end{array}$ & $\begin{array}{l}\text { Uninfected } \\
n=315(81.2 \%)\end{array}$ & & \\
\hline $\mathrm{RBC}$ & $(9-15) \times 10^{6} / \mathrm{LL}$ & $10.42 \pm 1.59$ & $9.71 \pm 1.31$ & $9.96 \pm 1.61$ & 0.710 & 0.494 \\
\hline $\mathrm{HCT}$ & $(27-45) \%$ & $31.44 \pm 3.87$ & $29.74 \pm 4.23$ & $29.74 \pm 4.43$ & 0.231 & 0.794 \\
\hline $\mathrm{Hb}$ & $(9-15) \mathrm{g} / \mathrm{dL}$ & $10.80 \pm 1.38$ & $10.19 \pm 1.40$ & $10.27 \pm 1.53$ & 0.255 & 0.775 \\
\hline WBC & $(4-8) \times 10^{3} \mu \mathrm{L}$ & $8.49 \pm 2.50$ & $9.76 \pm 2.74$ & $9.97 \pm 2.81$ & 1.845 & 0.159 \\
\hline Lymphocyte & $(2-9) \times 10^{3} \mu \mathrm{L}$ & $3.86 \pm 1.17$ & $4.34 \pm 1.41$ & $4.32 \pm 1.22$ & 2.134 & 0.120 \\
\hline Monocytes & $(0-0.75) \times 10^{3} \mu \mathrm{L}$ & $0.70 \pm 0.17$ & $0.79 \pm 0.25$ & $0.79 \pm 0.24$ & 1.246 & 0.289 \\
\hline Neutrophils & $(0.7-6.0) \times 10^{3} \mu \mathrm{L}$ & $2.94 \pm 1.02$ & $3.25 \pm 1.31$ & $3.42 \pm 1.47$ & 0.458 & 0.633 \\
\hline Eosinophils & $(0-1.0) \times 10^{3} \mu \mathrm{L}$ & $1.03 \pm 0.58$ & $1.28 \pm 0.78$ & $1.29 \pm 0.75$ & 0.205 & 0.814 \\
\hline Platelets & $(800-1100) \times 10^{3} \mu \mathrm{L}$ & $386.05 \pm 175.23$ & $333 \pm 141.08$ & $359.03 \pm 144.61$ & 0.555 & 0.575 \\
\hline
\end{tabular}

*Only two sheep harbored single $T$. ovis infection

sheep infested with ticks and tick-free sheep $(p=0.48)$ (Table 1) as well as animals with enlarged or nonenlarged lymph nodes $(p=0.28)$. However, the infection rate was significantly higher among exotic breeds $(36 \%$, $27 / 75$ ) (odds ratio 12.9 , CI 1.65-101.19) compared to indigenous $(16.2 \%, 47 / 291)$ and crossbreeds $(4.2 \%, 1 / 24)$ (odds ratio 2.9 CI 1.66-5.14) (Table 2).

Hematological indices including hematocrit, RBC counts, $\mathrm{Hb}, \mathrm{MCV}, \mathrm{MCHC}, \mathrm{WBC}$, eosinophils, and lymphocytes were not correlated with detection of Theileria infection. Nonetheless, compared to animals with mixed infections (T. lestoquardi plus T. ovis), sheep with single $T$. lestoquardi infections showed a lower average, but not significant values, of total WBCs $(8.49 \pm 2.5$ vs. $9.76 \pm 2.74, p>0.05)$, lymphocytes $(3.86 \pm 1.17$ vs. $4.34 \pm 1.41)$, monocytes $(0.70 \pm 0.17$ vs. $0.79 \pm 0.25)$, eosinophils $(1.03 \pm 0.58$ vs. $1.28 \pm 0.78)$, and neutrophils $(2.94 \pm 1.02$ vs. $3.25 \pm 1.31, p>0.05)$. However, all counts were within our normal clinical reference ranges (Table 3).

\section{Parasite density and clinical and hematological indices}

Total T. lestoquardi density among infected sheep, as judged by results of the PCR assay on blood samples, varied widely, between 1.03 and $5.99 \log _{10} 18 S$ rRNA copies $/ \mu \mathrm{L}$. The estimated mean density in single T. lestoquardi infection was higher (4.77 $\log _{10} 18 S$ rRNA copies/ $\mu \mathrm{L}$ blood, $95 \%$ CI $4.5-5.1 \log _{10}$ ) than mixed infection (4.2 $\log _{10} 18 S r R N A$ copies/ $\mu \mathrm{L}$ blood, 95\% CI 3.9-3.5 $\left.\log _{10}\right)$, but this difference was not significant $(p=0.12)$. Similarly, T. ovis density among the infected sheep varied widely, between 1.06 and $5.30 \log 1018 S$ rRNA copies $/ \mu \mathrm{L}$ (mean $=4.03)$. Single T. ovis, detected in only two samples, had a higher average density of $4.3 \log _{10} 18 \mathrm{~S}$ $r R N A$ copies $/ \mu \mathrm{L}$ blood $\left(95 \%\right.$ CI $\left.3.21-5.37 \log _{10}\right)$, than the mean density of $T$. ovis among mixed infection (4.02, $\log _{10} 18 S r R N A$ copies/ $\mu \mathrm{L}$ blood, 95\% CI 3.84-4.22 $\log _{10}$ ). In mixed infections, T. lestoquardi was present at a slightly higher density $\left(4.2 \log _{10} 18 S\right.$ rRNA copies $/ \mu \mathrm{L}$ blood, $95 \%$ CI $\left.3.9-4.5 \log _{10}\right)$ than T. ovis (4.02 $\log _{10} 18 S$ rRNA copies/ $\mu \mathrm{L}$ blood, 95\% CI 3.8-4.2 $\log _{10}$ ) $(p=0.184)$. Nonetheless, there was a highly significant association between the densities of the two species in mixed infection in individual animals $(p<0.0001)$.

Density of both T. lestoquardi and T. ovis was not associated with gender $(p>0.25)$, breed $(p>0.47)$, presence of ticks $(p>0.25)$ or enlargement of lymph nodes $(p>0.45)$. Moreover, T. lestoquardi, when present as a single infection, had no significant effect on any of the examined hematological indices $(p>0.05)$.

\section{Genetic diversity and structure of $T$. lestoquardi}

Seventy-three T. lestoquardi isolates from Barka were successfully genotyped for ten species-specific microsatellites. Eight carried T. lestoquardi but not T. ovis and 65 harbored mixed species (T. lestoquardi plus T. ovis).

The total number of alleles detected for each of the $T$. lestoquardi loci ranged from 5 for TL_MS25 to 22 for TL_MS280, and the number of alleles per locus within a single sample ranged from 1 to 6 . A high level of polymorphism was observed among six loci (TL_MS205, TL_MS281, TL_MS280, TL_MS07, TL_MS13, and TL_ MS19) while a moderate level of diversity was observed for a further 4 loci (TL_MS04, TL_MS16, TL_MS18, and TL_MS25) (Table 4). The average heterozygosity among single T. lestoquardi infections was significantly 
Table 4 Allelic diversity and unbiased heterozygosity (He) at 10 micro- and mini-satellite loci among 73 T. lestoquardi isolates collected in Barka in 2019 and 190 isolates examined in 2014 in different regions in Oman

\begin{tabular}{|c|c|c|c|c|c|c|c|c|c|c|c|}
\hline Population & $\begin{array}{l}\mathrm{TL}_{-} \\
\mathrm{MS05}\end{array}$ & $\begin{array}{l}\mathrm{TL} \\
\mathrm{MS} 281\end{array}$ & $\begin{array}{l}\text { TL } \\
\text { MS04 }\end{array}$ & $\begin{array}{l}\mathrm{TL} \\
\mathrm{MS} 07\end{array}$ & $\begin{array}{l}\mathrm{TL}_{-} \\
\mathrm{MS} 13\end{array}$ & $\begin{array}{l}\mathrm{TL} \\
\mathrm{MS} 16\end{array}$ & $\begin{array}{l}\text { TL } \\
\text { MS19 }\end{array}$ & $\begin{array}{l}\text { TL } \\
\text { MS280 }\end{array}$ & $\begin{array}{l}\text { TL } \\
\text { MS25 }\end{array}$ & $\begin{array}{l}\mathrm{TL} \\
\mathrm{MS18}\end{array}$ & Average \\
\hline $2019(n=73)$ & 0.861 & 0.737 & 0.673 & 0.857 & 0.925 & 0.594 & 0.831 & 0.893 & 0.579 & 0.662 & 0.772 \\
\hline $2014(n=190)$ & 0.867 & 0.839 & 0.359 & 0.705 & 0.796 & 0.441 & 0.665 & 0.760 & 0.121 & 0.548 & 0.582 \\
\hline
\end{tabular}

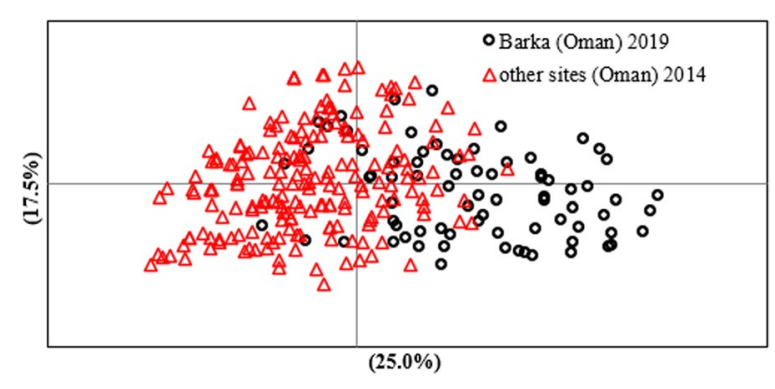

Fig. 2 Principal coordinate analysis (PCOA) of T. lestoquardi populations from Barka and other regions in Oman collected on 2014 PCoA was performed on the multi-locus genotype data representing each of the populations sampled. The proportion of the variation in the dataset explained by each axis is indicated in parentheses

lower $\left(H_{e}=0.685\right)$, ranging from 0.00 to 0.893 , than that in mixed ( $T$. lestoquardi/T. ovis) infections $\left(H_{e}=0.76\right)$, ranging from 0.590 to $0.933(p=0.0001)$.

\section{Multiplicity of infection}

Seventy-two (98.7\%) of the above 73 T. lestoquardi samples carried multiple parasite genotypes. The high prevalence of genotype multiplicity in Barka (98.7\%) is consistent with that seen in other sites in 2014 (96\%) in Oman. The extent of multiplicity and the number of genotypes per sample were slightly lower among single T. lestoquardi infection, ranging between 2 and 5, mean 3.4 ( $\mathrm{SD} \pm 0.92)$, compared to mixed (T. lestoquardi/T. ovis) infection, ranging between 1 to 6 , mean 3.59 $(\mathrm{SD} \pm 1.04)$.

\section{Linkage disequilibrium}

LD was estimated using the standard index of association to investigate whether the high diversity observed in T. lestoquardi in Barka could be explained by a panmictic population structure and high rates of recombination in the tick vector. When all samples collected in all farms were treated as a single population, an $\mathrm{I}^{\mathrm{S}}{ }_{\mathrm{A}}$ value of (0.027) and a $V_{D}$ value (2.06) greater than $L$ (1.97) was obtained, indicating LD.

\section{Population sub-structuring}

$\mathrm{F}_{\mathrm{ST}}$ values $\left(\mathrm{G}_{\mathrm{ST}}^{\prime}\right.$ and $\left.\theta\right)$ were estimated to measure the level of genetic relatedness of T. lestoquardi in Barka in 2019 compared to a previous dataset from parasites isolated in different sites in Oman in 2014 employing the same panel of markers [9].

No differentiation was observed between the T. lestoquardi population samples collected in different sites in Oman in $2014\left(G_{\mathrm{ST}}^{\prime}=0.024\right.$ and $\left.\theta=0.024\right)$. However, a moderate level of differentiation $\left(G_{\mathrm{ST}}^{\prime}=0.096\right.$ and $\theta=0.04$ ) was observed between parasite populations in Oman in 2014 and Barka in 2019. The estimated differentiation is supported by principal coordinate analysis (PCoA) (Fig. 2). PCoA demonstrated evidence of structuring, with some haplotypes distributed independently of year of collection. Haplotypes from 2014 and 2019 are overlapped, however, a few lineages from 2019 diverge from the rest of individuals.

\section{Discussion}

The present study provides additional evidence for within-host interaction of $T$. ovis and T. lestoquardi in small ruminants in Oman [10]. The density of both parasites was higher when present as a single species infection (presence of one species alone), compared to mixed-species infections (presence of the two species), which we believe provides support for the concept of heterologous protection, the ability to limit parasite load conferred by mixed infection with both parasites [14]. This was partly reflected as a disproportionately higher prevalence of mixed infection (T. lestoquardi and T. ovis) among animals, compared to single infection with each, supporting the hypothesis of heterologous protection against disease in endemic areas [14]. This is consistent with epidemiological surveys in a different region in Oman, where mixed infection with T. lestoquardi and T. ovis in sheep was remarkably higher than single infection of each species separately [5]. However, there is information on the extent of mixed-species infection in the main tick vector in the region, Hyalomma anatolicum.

Within-host interaction between $T$. ovis and T. lestoquardi is a reasonable explanation for the occurrence of T. lestoquardi (pathogenic parasite) at a lower density when present as a mixed infection (4.2 $\log _{10} 18 S$ rRNA copies/ $\mu \mathrm{L}$ blood, 95\% CI 3.9-3.5 $\log _{10}$ ) compared to 
single infection (4.77 $\log _{10} 18 S r R N A$ copies/ $\mu \mathrm{L}$ blood, 95\% CI 4.5-5.1 $\left.\log _{10}\right)$, though this difference was not significant $(p=0.12)$. Furthermore, the pathogenic parasite (T. lestoquardi) was present at a relatively higher density (4.2 $\log _{10} 18 S r R N A$ copies/ $\mu \mathrm{L}$ blood, 95\% CI 3.9-4.5 $\log _{10}$ ) than the non-pathogenic (T. ovis) (4.02 $\log _{10} 18 \mathrm{~S}$ rRNA copies/ $\mu \mathrm{L}$ blood, 95\% CI 3.8-4.2 $\left.\log _{10}\right)(p=0.184)$. Unfortunately, few animals displayed single $T$. ovis infection $(n=2)$. However, in those that showed single T. ovis, the parasitemia was always relatively higher compared to mixed infections (4.3 vs. $4.02 \log _{10} 18 S r R N A$ copies $/ \mu \mathrm{L}$ ). The lower T. lestoquardi parasitemia in mixed infection is indicative of immune pressure (potentially innate), which is expected to impact both parasites (T. lestoquardi and $T$. ovis). The above findings are in line with our most recent data in a cohort study of indigenous sheep in Oman, which revealed a significantly higher T. lestoquardi density in single infection versus mixed infection [10].

The observation of predominantly mixed-species infection (86.7\%) among the asymptomatic animals examined in the present study is in line with the hypothesis of heterologous protection against disease in endemic areas [14]. Mixed-species infection of some apicomplexan parasites, as well as co-existence of multiple genotypes of the same species, have been considered advantageous to the host, often associated with reduced morbidity and mortality [27]. Evidence for a protective effect of mixed Theileria spp., in mortality of East Coast fever due to $T$. parva infection, in the presence of less pathogenic Theileria spp. (T. mutans and T. velifera) has been demonstrated in indigenous African Bos indicus calves [14]. This is consistent with our most recent work in Oman that linked mixed infection (T. lestoquardi plus T. ovis) to significant reduction of mortality among local breeds of sheep [10]. The present study extended these earlier findings and examined clinical and hematological indices in sheep carrying mixed (T. lestoquardi plus T. ovis) compared to single T. lestoquardi infections. Lower levels of WBCs, lymphocytes, and neutrophils were seen among single T. lestoquardi infection compared to mixed infection, however, these differences were not associated with overt clinical signs and were not statistically significant (Table 3). The limited proportion of animals with single T. lestoquardi infection (10.6\%) in the present study and related potential variation caused by other pathogens in hematological indices precluded statistical validation of the impact of type of infection on some morbidity markers. Thus, further studies to substantiate these findings would require data from a larger sample of animals infected with single infections (T. lestoquardi and T. ovis) to obtain statistically valid comparisons.

A high level of genetic diversity and genotype multiplicity of T. lestoquardi was detected in infected sheep in Barka. However, no differences in the extent of diversity were seen among parasites causing single $(\mathrm{He}=0.68)$ versus mixed infection $(H e=0.76)$. Similarly, multiple genotypes were common among single and mixed $T$. lestoquardi infections with equal extent of multiplicity suggesting a common mechanism for regulation of the dynamics of genotypes. The matched level of $T$. lestoquardi diversity detected in animals with single and mixed-species infection suggests that both forms of infection are equally susceptible to establishment of multiple genotype populations. This can be attributed to high abundance and infestation of $H$. anatolicum, which can promote cross-mating and recombination within ticks to generate novel parasite genotypes for infection of sheep. The above is consistent with the high genetic diversity and multiplicity of infection reported for T. lestoquardi populations in Oman [9] and Sudan [28].

Very low levels of genetic differentiation were detected between T. lestoquardi parasites collected in 2014 in different sites in Oman, including Batinah where the current study was carried out, with an average pairwise $F_{\mathrm{ST}}$ value of 0.024 . This is consistent with the high rate of tick infestation among the infected sheep, which can lead to a high level of cross-mating and recombination in ticks, to generate novel genotypes. However, a moderate level of genetic differentiation was measured between $T$. lestoquardi parasites in Barka examined in the current study in 2019 and parasites in other sites in Oman, with $F_{\mathrm{ST}}$ values of 0.048 , though, complete separation between the parasite populations was not observed (Fig. 2). This moderate level of differentiation is consistent with the data from similar analysis between $T$. annulata in Tunisia and Turkey $\left(F_{\mathrm{ST}}\right.$ values of 0.049) [29]. A more profound genetic differentiation has been observed between $T$. annulata and T. lestoquardi in Oman and populations in widely separated countries, consistent with geographical and trade barriers hindering gene flow $[9,21,28]$. For example, a high level of differentiation $\left(F_{\mathrm{ST}}=0.295\right)$ was evident between T. lestoquardi populations in Oman and Sudan, while no temporal differentiation was observed between T. lestoquardi population collected in Sudan in 2013 compared to $2016\left(F_{\mathrm{ST}}=0.29\right)$ [28]. Unlike the findings in Sudan, the present study revealed temporal moderate differentiation between parasites in Barka (2019) and other sites in Oman (2014), which is likely to be due to trade and importation of asymptomatic parasite carriers from outside the country through commercial trade of sheep, allowing admixture and intercrossing with local parasite populations in Oman. This is consistent with observations of similar patterns of admixed genetic structure of populations of livestock in Oman, cattle and goats, with African and Near East origin [30, 31], facilitating dissemination of vector-borne parasites via trade 
[32]. A large proportion of the sheep examined (19.2\%) in the present study were of exotic breed, which represents a large proportion of imported animals (Table 1) that frequently are carriers of $T$. lestoquardi. In March 2020, Oman imported 138,200 head of sheep, and 8427 head of cattle from different parts of the world, including Australia, Sudan, South Africa, and Somalia [33]. Animal movement between countries poses a significant threat for dissemination of parasites and their vectors. In turn, this can lead to a high level of gene flow between parasite populations from close geographical locations.

In summary, the present study provides additional evidence of within-host interaction between the T. lestoquardi and T. ovis, and noticeable differences in some hematological indices and parasite density among single T. lestoquardi infection compared to mixed infection. The extent of genetic diversity of $T$. lestoquardi that exists as single infection is similar to that in mixed infection suggesting that the parasites in the two groups are homogenous. The T. lestoquardi genotypes detected in Barka in 2019 showed moderate differentiation compared to those seen in other sites in Oman in 2014, suggesting that new parasite strains may have been introduced in the area via importation of infected animals.

\begin{abstract}
Abbreviations
GCC: Gulf Cooperation Council; MOT: Malignant ovine theileriosis; RBC: Red blood cells; HCT: Hematocrit; Hb: Hemoglobin; MCV: Mean corpuscular volume; MCHC: Mean corpuscular hemoglobin concentration; WBC: Total white blood cell count; PCR: Polymerase chain reaction; qPCR: Quantitative polymerase chain reaction; MOI: Multiplicity of infection; $\mathrm{VD}$ : Variance of mismatch values; LD: Linkage disequilibrium; PCOA: Principal coordinate analysis; He: Heterozygosity; $\left.\right|_{A} ^{S}$ : Association of index; $F_{S T}$ : Wright's fixation index.
\end{abstract}

\section{Acknowledgements}

We are grateful to the farmers and the staff of Al-Falahi Clinic, Barka, Oman, for their support with field surveys. We appreciate the support of the technical staff of the Department of Animal and Veterinary Sciences, College of Agricultural and Marine Sciences and Biochemistry Department, College of Medicine and Health Sciences, Sultan Qaboos University, Oman.

\section{Authors' contributions}

$H A B, I M$, and $E J$ conceived the study and designed the experiments. EE and SA contributed to data collection and data analysis. All authors read and approved the final manuscript.

\section{Funding}

The project is supported by The Research Council Oman (TRC, Oman) project RC/MED/BIOC/13/01.

\section{Availability of data and materials}

All data generated or analyzed during this study are included in this published article.

\section{Declarations}

\section{Ethics approval and consent to participate}

The research was performed in accordance with the relevant guidelines and regulations of the ethical and animal welfare code of Sultan Qaboos University, Oman and the Ministry of Agriculture and Fisheries, Oman. Oral consent was obtained from the farm owner before drawing blood from animals.

\section{Consent for publication}

Not applicable.

\section{Competing interests}

The authors declare that they have no competing interests with regard to this publication.

\section{Author details}

${ }^{1}$ Department of Biochemistry, College of Medicine and Health Sciences, Sultan Qaboos University, AlKhoud 123, PO Box 35, Muscat, Oman. ${ }^{2}$ Department of Animal and Veterinary Sciences, College of Agricultural and Marine Sciences, Sultan Qaboos University, Muscat, Oman. ${ }^{3}$ Central Veterinary Research Laboratories, Al Amarat, P.O. Box 8067, Khartoum, Sudan. ${ }^{4}$ Laboratory for Hormozgan Veterinary, Bandar Abbas, Iran. ${ }^{5}$ Division of Infection and Immunity, The Roslin Institute, Royal (Dick) School of Veterinary Studies, University of Edinburgh, Edinburgh, UK. ${ }^{6}$ Institute of Immunology and Infection Research, School of Biological Sciences, University of Edinburgh, Edinburgh, UK.

Received: 28 February 2021 Accepted: 26 June 2021

Published online: 20 July 2021

\section{References}

1. Altay K, Dumanli N, Aktas M. A study on ovine tick-borne hemoprotozoan parasites (Theileria and Babesia) in the East Black Sea Region of Turkey. Parasitol Res. 2012;111(1):149-53.

2. Panel, Ahaw. Scientific opinion on geographic distribution of tick-borne infections and their vectors in Europe and the other regions of the Mediterranean Basin. EFSA J. 2010;2010(8):1723.

3. Yin H, Schnittger L, Luo J, Seitzer U, Ahmed JS. Ovine theileriosis in China: a new look at an old story. Parasitol Res. 2007;101(2):191-5.

4. Tageldin MH, Fadiya AAK, Sabra AAY, Ismaily SIAI. Theileriosis in sheep and goats in the Sultanate of Oman. Trop Anim Health Prod. 2005;37(6):491-3. https://doi.org/10.1007/s11250-005-2475-4.

5. Al-Fahdi A, Alqamashoui B, Al-Hamidhi S, Kose O, Tageldin MH, Bobade $P$, et al. Molecular surveillance of Theileria parasites of livestock in Oman. Ticks Tick-borne Dis. 2017;8(5):741-8.

6. Uilenberg G. Immunization against diseases caused by Theileria parva: a review. Tropical Med Int Health. 1999;4(9):A12-20.

7. Gharbi M, Touay A, Khayeche M, Laarif J, Jedidi M, Sassi L, et al. Ranking control options for tropical theileriosis in at-risk dairy cattle in Tunisia, using benefit-cost analysis. Revue Scientifique et Technique-OIE. 2011;30(3):763

8. Al-Fahdi A. Molecular identification and phylogenetic studies of theileria parasite in Oman. Muscat: Sultan Qaboos University; 2015.

9. Al-Hamidhi S, Weir W, Kinnaird J, Tageledin M, Beja-Pereira A, Morrison I, et al. Theileria lestoquardi displays reduced genetic diversity relative to sympatric Theileria annulata in Oman. Infect Genet Evol. 2016;43:297306. https://doi.org/10.1016/j.meegid.2016.05.007.

10. Awad H, Gadalla AA, Postigo M, Al-Hamidhi S, Tageldin MH, Skariah S, et al. Dynamics and within-host interaction of Theileria lestoquardi and $T$. ovis among naive sheep in Oman. Sci Rep. 2020;10(1):1-9.

11. Bruce MC, Donnelly CA, Alpers MP, Galinski MR, Barnwell JW, Walliker D, et al. Cross-species interactions between malaria parasites in humans. Science. 2000:287(5454):845-8.

12. Telfer $S$, Lambin $X$, Birtles $R$, Beldomenico P, Burthe $S$, Paterson $S$, et al. Species interactions in a parasite community drive infection risk in a wildlife population. Science. 2010;330(6001):243-6.

13. Purnell R, Young E, Brocklesby D, Hendry D. The haematology of experimentally-induced B divergens and E phagocytophila infections in splenectomised calves. Vet Rec. 1977;100(1):4-6.

14. Woolhouse ME, Thumbi SM, Jennings A, Chase-Topping M, Callaby R, Kiara $\mathrm{H}$, et al. Co-infections determine patterns of mortality in a population exposed to parasite infection. Sci Adv. 2015;1 (2):e1400026.

15. Ayadi O, Gharbi M, Benchikh-Elfegoun MC. Haematological and biochemical indicators of tropical theileriosis diseased cattle in wilaya of Sétif (North East Algeria). J Parasit Dis. 2017;41 (2):538-42.

16. Mahmmod YS, Elbalkemy FA, Klaas IC, Elmekkawy MF, Monazie AM. Clinical and haematological study on water buffaloes (Bubalus bubalis) 
and crossbred cattle naturally infected with Theileria annulata in Sharkia province, Egypt. Ticks Tick-borne Dis. 2011;2(3):168-71.

17. Singh SK, Roeffen W, Mistarz UH, Chourasia BK, Yang F, Rand KD, et al. Construct design, production, and characterization of Plasmodium falciparum 48/45 R0. 6C subunit protein produced in Lactococcus lactis as candidate vaccine. Microb Cell Fact. 2017;16(1):1-11.

18. Razmi G, Yaghfoori S, Mohri M, Haghparast A, Tajeri S. The haematological, proinflammatory cytokines and lgG changes during an ovine experimental theileriosis. Onderstepoort J Vet Res. 2019;86(1):1-6.

19. Sandhu G, Grewal A, Singh A, Kondal J, Singh J, Brar R. Haematological and biochemical studies on experimental Theileria annulata infection in crossbred calves. Vet Res Commun. 1998;22(5):347-54.

20. Yaghfoori S, Mohri M, Razmi G. Experimental Theileria lestoquardi infection in sheep: biochemical and hematological changes. Acta Trop. 2017;173:55-61.

21. Al-Hamidhi S, Tageldin MH, Weir W, Al-Fahdi A, Johnson EH, Bobade P, et al. Genetic diversity and population structure of Theileria annulata in Oman. PLoS ONE. 2015;10(10):e0139581.

22. Anderson TJ, Haubold B, Williams JT, Estrada-Franco JG, Richardson L, Mollinedo $R$, et al. Microsatellite markers reveal a spectrum of population structures in the malaria parasite Plasmodium falciparum. Mol Biol Evol. 2000;10:1467-82.

23. Peakall R, Smouse P. GenAIEx 6.5: genetic analysis in Excel. Population genetic software for teaching and research—an update. Bioinformatics. 2012;28:2537-9.

24. Anon A. 1996. The Evaluation of Forensic DNA Evidence. Washington, DC: National Academy Press.
25. Weir BS, Cockerham CC. 1984. Estimating F-statistics for the analysis of population structure. evolution, pp.1358-1370.

26. Haubold B, Hudson RR. LIAN 3.0: detecting linkage disequilibrium in multilocus data. Bioinformatics. 2000;16(9):847-9.

27. Smith T, Felger I, Kitua A, Tanner M, Beck HP. 7. Dynamics of multiple Plasmodium falciparum infections in infants in a highly endemic area of Tanzania. Trans R Soc Trop Med Hyg. 1999;93(1):35-9.

28. Awad H, Al-Hamidhi S, El Hussein ARM, Zein Yousif YM, Taha KM, Salih DA, et al. Theileria lestoquardi in Sudan is highly diverse and genetically distinct from that in Oman. Infect Genet Evol. 2018;62:46-52.

29. Weir W, Ben-Miled L, Karagenç T, Katzer F, Darghouth M, Shiels B, et al. Genetic exchange and sub-structuring in Theileria annulata populations. Mol Biochem Parasitol. 2007;154(2):170-80.

30. Al-Araimi NA, Al-Atiyat RM, Luzuriaga-Neira A, Gaafar OM, Kadim IT, Al-Marzoogi W, et al. Genetic structure of Omani goats reveals admixture among populations from geographically proximal sites. Small Rumin Res. 2019;178:1-6.

31. Mahgoub O, Babiker HA, Kadim I, Al-Kindi M, Hassan S, Al-Marzoogi W, et al. Disclosing the origin and diversity of O mani cattle. Anim Genet. 2013:44(3):336-9.

32. Barré N, Uilenberg G. Spread of parasites transported with their hosts: case study of two species of cattle tick. Rev Sci Tech. 2010;29(1):149.

33. Daily M: Over $1 \mathrm{mn}$ livestock imported in 7 months; 2020.

\section{Publisher's Note}

Springer Nature remains neutral with regard to jurisdictional claims in published maps and institutional affiliations.
Ready to submit your research? Choose BMC and benefit from:

- fast, convenient online submission

- thorough peer review by experienced researchers in your field

- rapid publication on acceptance

- support for research data, including large and complex data types

- gold Open Access which fosters wider collaboration and increased citations

- maximum visibility for your research: over $100 \mathrm{M}$ website views per year

At BMC, research is always in progress.

Learn more biomedcentral.com/submissions 